Commun. math. Phys. 39, 91-110 (1974)

(C) by Springer-Verlag 1974

\title{
Markovian Master Equations
}

\author{
E. B. Davies \\ Mathematical Institute, Oxford, England
}

Received June 6, 1974

\begin{abstract}
We give a rigorous proof that under certain technical conditions the memory effects in a quantum-mechanical master equation become negligible in the weak coupling limit. This is sufficient to show that a number of open systems obey an exponential decay law in the weak coupling limit for a rescaled time variable. The theory is applied to a fairly general finite dimensional system weakly coupled to an infinite free heat bath.
\end{abstract}

\section{§ 1. Introduction}

In the last fifteen years there has been a growing realisation by physicists of the importance of master equations for the study of the time evolution of open quantum-mechanical systems. As well as providing a suitable framework for the consideration of the fundamental property of irreversibility [1], they have proved an important technique in the analysis of a variety of models, such as harmonic oscillators and lasers. It becomes clear in the excellent survey article of Haake [2] that one of the main reasons for the usefulness of master equations is the radical simplification obtained when memory effects are neglected.

It is rather surprising, therefore, that in the recent rigorous studies of these models, the use of master equations has been avoided. This appears to be because, although it is possible to give a rigorous proof of the master equation itself, conditions under which the memory effects can be neglected have not been found.

In this paper we give a rigorous proof that the time evolution of an open system is Markovian in the weak coupling limit. As the coupling constant converges to zero we rescale the time variable to compensate for the slower decay of the system. The theory is developed in a general form and its application to a variety of models is outlined. The case of a general finite-dimensional system weakly coupled to an infinite free heat bath is investigated in some detail and relaxation to the Gibbs state is proved. 


\section{§ 2. The Abstract Theory of the Weak Coupling Limit}

In order to establish our notation we start with the derivation of the master equation. For motivation and a historical discussion we refer the reader to $[1,2]$. We let $P_{0}$ be a projection on a Banach space $\mathscr{B}$ and put $P_{1}=1-P_{0}$. We refer to $\mathscr{B}_{0}=P_{0} \mathscr{B}$ as the system and to $\mathscr{B}_{1}$ $=P_{1} \mathscr{B}$ as the bath. We suppose that the free evolution of both is determined by a strongly continuous one-parameter group $U_{t}$ of isometries on $\mathscr{B}$ which leaves each of $\mathscr{B}_{0}, \mathscr{B}_{1}$ invariant. The infinitesimal generator $Z$ is then closed and densely defined and

$$
\left[Z, P_{0}\right]=0 .
$$

We define $Z_{i}=P_{i} Z$ for later use.

We introduce a perturbation $A$ which is supposed to be a bounded operator on $\mathscr{B}$. Writing $A_{i j}=P_{i} A P_{j}$, we suppose from here onwards that

$$
A_{00}=0 \text {. }
$$

We let $U_{t}^{\lambda}$ be the one-parameter group generated by $Z+\lambda A_{11}$ so that for all $t$

$$
\left[U_{t}^{\lambda}, P_{0}\right]=0 .
$$

We also let $V_{t}^{\lambda}$ be the one-parameter group generated by $Z+\lambda A$, so that by a well-known formula [3]

$$
V_{t}^{\lambda}=U_{t}^{\lambda}+\lambda \int_{s=0}^{t} U_{t-s}^{\lambda}\left(A_{01}+A_{10}\right) V_{s}^{\lambda} d s .
$$

In this and all subsequent integrals, the integrand is everywhere bounded and strongly continuous, so no difficulties of interpretation occur. From Eq. (2.4) we can obtain

and

$$
P_{0} V_{t}^{\lambda} P_{0}=U_{t}^{\lambda} P_{0}+\lambda \int_{s=0}^{t} U_{t-s}^{\lambda} A_{01} P_{1} V_{s}^{\lambda} P_{0} d s
$$

$$
P_{1} V_{t}^{\lambda} P_{0}=\lambda \int_{s=0}^{t} U_{t-s}^{\lambda} A_{10} P_{0} V_{s}^{\lambda} P_{0} d s .
$$

Putting $W_{t}^{\lambda}=P_{0} V_{t}^{\lambda} P_{0}$ we obtain by substitution

$$
W_{t}^{\lambda}=U_{t}^{\lambda} P_{0}+\lambda^{2} \int_{s=0}^{t} \int_{u=0}^{s} U_{t-s}^{\lambda} A_{01} U_{s-u}^{\lambda} A_{10} W_{u}^{\lambda} d u d s .
$$


Using Eq. (2.2) we finally obtain

$$
W_{t}^{\lambda}=U_{t}+\lambda^{2} \int_{s=0}^{t} \int_{u=0}^{s} U_{t-s} A_{01} U_{s-u}^{\lambda} A_{10} W_{u}^{\lambda} d u d s
$$

where we have dropped reference to $P_{0}$ since we shall from now on work entirely within $\mathscr{B}_{0}$.

This is an integrated form of the master equation constructed by Nakajima, Prigogine, Resibois, and Zwanzig. To see this we put $\varphi_{t}=W_{t}^{\lambda} \varphi$ where $\varphi \in \mathscr{B}_{0}$ to get

$$
\varphi_{t}=U_{t}\left(\varphi_{0}+\lambda^{2} \int_{s=0}^{t} \int_{u=0}^{s} U_{-s} A_{01} U_{s-u}^{\lambda} A_{10} \varphi_{u} d u d s\right)
$$

so that formally

$$
\frac{\partial \varphi_{t}}{\partial t}=Z_{0} \varphi_{t}+\lambda^{2} \int_{u=0}^{t} A_{01} U_{t-u}^{\lambda} A_{10} \varphi_{u} d u .
$$

However we prefer not to work with this equation because it necessitates consideration of domain questions. It does indicate that in Eq. (2.8) the integral contains memory terms. We now come to the problem of going to the weak coupling limit.

Since the memory term is small compared with the free term we change to the interaction representation before letting $\lambda \rightarrow 0$. Putting

$$
Y_{\tau}^{\lambda}=U_{-t} W_{t}^{\lambda}
$$

where $\tau=\lambda^{2} t$ we obtain

where

$$
Y_{\tau}^{\lambda}=1+\int_{\sigma=0}^{\tau} H(\lambda, \tau-\sigma, \sigma) Y_{\sigma}^{\lambda} d \sigma
$$

and

$$
H(\lambda, \tau, \sigma)=U_{-\lambda-2 \sigma} K(\lambda, \tau) U_{\lambda-2 \sigma}
$$

$$
K(\lambda, \tau)=\int_{x=0}^{\lambda-2 \tau} U_{-x} A_{01} U_{x}^{\lambda} A_{10} d x
$$

If the kernel $H(\lambda, \tau, \sigma)$ converges as $\lambda \rightarrow 0$ to an operator $H$ on $\mathscr{B}_{0}$ which is independent of $\tau$ and $\sigma$ then the convergence of $V_{\tau}^{\lambda}$ to a limit operator $Y_{\tau}$ as $\lambda \rightarrow 0$ may easily be proved. However upon examining the dependence of $H(\lambda, \tau, \sigma)$ on $\sigma$ it becomes apparent that such limiting behaviour is unlikely, and that a more sophisticated approach is necessary. 
We now restrict attention to the case where $\mathscr{B}_{0}$ is finite dimensional. If $\left\{Q_{\alpha}\right\}$ are the spectral projections of $Z_{0}$ on $\mathscr{B}_{0}$ then we can write

$$
P_{0} U_{t}=e^{Z_{0} t}=\sum_{\alpha} Q_{\alpha} e^{i \omega_{\alpha} t}
$$

where the $\omega_{\alpha}$ are distinct and real. If $X$ is any operator on $\mathscr{B}_{0}$ we define

$$
X^{\natural}=\sum_{\alpha} Q_{\alpha} X Q_{\alpha}
$$

or, equivalently

$$
X^{\natural}=\lim _{t \rightarrow \infty} \frac{1}{2 t} \int_{-t}^{t} U_{x} X U_{-x} d x
$$

which makes no reference to the spectral projections of $Z_{0}$.

Theorem 2.1. Suppose that for all $\tau_{1}>0$ there is a constant $c$ such that

$$
\|K(\lambda, \tau)\| \leqq c
$$

provided $|\lambda| \leqq 1$ and $0 \leqq \tau \leqq \tau_{1}$. Suppose also that there is a bounded operator $K$ on $\mathscr{B}_{0}$ such that if $0<\tau_{0} \leqq \tau_{1}<\infty$ then

$$
\lim _{\lambda \rightarrow 0}\|K(\lambda, \tau)-K\|=0
$$

uniformly with respect to $\tau$ if $\tau_{0} \leqq \tau \leqq \tau_{1}$. Then if $\mathscr{B}_{0}$ is finite-dimensional and $b \in \mathscr{B}_{0}$

$$
\lim _{\lambda \rightarrow 0}\left\|Y_{\tau}^{\lambda} b-Y_{\tau} b\right\|=0
$$

uniformly on each interval $0 \leqq \tau \leqq \tau_{1}$, where

where

$$
Y_{\tau}=\exp \left\{K^{\natural} \tau\right\}
$$

$$
K^{\natural} U_{t}=U_{t} K^{\natural}
$$

as operators on $\mathscr{B}_{0}$ for all $t \in \mathbb{R}$.

Proof. We let $\mathscr{V}$ be the Banach space of continuous $\mathscr{B}_{0}$-valued functions on $\left[0, \tau_{1}\right]$. If $\mathscr{H}_{\lambda}: \mathscr{V} \rightarrow \mathscr{V}$ is defined by

$$
\left(\mathscr{H}_{\lambda} f\right)(\tau)=\int_{\sigma=0}^{\tau} H(\lambda, \tau-\sigma, \tau) f(\sigma) d \sigma
$$

then $f_{\lambda}=Y_{\tau}^{\lambda} b$ is the solution of

$$
f_{\lambda}=g+\mathscr{H}_{\lambda} f_{\lambda}
$$


where $g(\tau)=b$ for $0 \leqq \tau \leqq \tau_{1}$. We now define $\tilde{\mathscr{H}}_{\lambda}: \mathscr{V} \rightarrow \mathscr{V}$ by

$$
\left(\tilde{\mathscr{H}}_{\lambda} f\right)(\tau)=\int_{\sigma=0}^{\tau} U_{-\lambda^{-2} \sigma} K U_{\lambda^{-2} \sigma} f(\sigma) d \sigma
$$

By Eqs. (2.13) and (2.19) it is easy to show that $\left(\mathscr{H}_{\lambda}-\tilde{\mathscr{H}}_{\lambda}\right)$ converges strongly to zero as $\lambda \rightarrow 0$.

Using Eq. (2.15) we can put

$$
\left(\tilde{\mathscr{H}}_{\lambda} f\right)(\tau)=\sum_{\alpha, \beta} Q_{\beta} K Q_{\alpha} \int_{\sigma=0}^{\tau} e^{i\left(\omega_{\alpha}-\omega_{\beta}\right) \lambda-2 \sigma} f(\sigma) d \sigma .
$$

As $\lambda \rightarrow 0$ this converges uniformly for $0 \leqq \tau \leqq \tau_{1}$ to

$$
h(\tau)=\sum_{\alpha, \beta} Q_{\beta} K Q_{\alpha} \delta_{\alpha \beta} \int_{\sigma=0}^{\tau} f(\sigma) d \sigma .
$$

Therefore $\tilde{\mathscr{K}}_{\lambda}$, and hence $\mathscr{K}_{\lambda}$, converges strongly to $\mathscr{K}: \mathscr{V} \rightarrow \mathscr{V}$ where

$$
(\mathscr{K} f)(\tau)=\int_{\sigma=0}^{\tau} K^{\natural} f(\sigma) d \sigma .
$$

Now $f(\tau)=Y_{\tau} b$ is the solution of

so

$$
f=g+\mathscr{K} f
$$

$$
f=g+\mathscr{K} g+\mathscr{K}^{2} g+\cdots
$$

and a similar equation holds for $f_{\lambda}$. Therefore

$$
\left\|f_{\lambda}-f\right\| \leqq \sum_{n=1}^{\infty}\left\|\mathscr{K}_{\lambda}^{n} g-\mathscr{K}^{n} g\right\| .
$$

Since $\mathscr{K}_{\lambda}$ converges strongly to $\mathscr{K}$ each term of this series converges to zero. Also since $\mathscr{K}_{\lambda}$ and $\mathscr{K}$ are Volterra integral operators

$$
\left\|\mathscr{K}_{\lambda}^{n} g-\mathscr{K}^{n} g\right\| \leqq 2\|g\| c^{n} \tau_{1}^{n} / n !
$$

Therefore $f_{\lambda}$ converges in norm to $f$ as $\lambda \rightarrow 0$.

A simple condition for the existence of the limit operator is given below.

Theorem 2.2. If $A_{11}=0$ and

$$
\int_{0}^{\infty}\left\|A_{01} U_{x} A_{10}\right\| d x<\infty
$$

then the conditions of Theorem 2.1 are satisfied with

$$
K=\int_{0}^{\infty} U_{-x} A_{01} U_{x} A_{10} d x
$$


Proof. This is immediate once it is realized that when $A_{00}=A_{11}=0$, the operator $U_{t}^{\lambda}$ is independent of $\lambda$.

In order to deal with the more physically interesting case where $A_{11} \neq 0$ we introduce the notation

$$
A_{r}=U_{-t_{r}} A U_{t_{r}} .
$$

Theorem 2.3. Suppose that

Defining

$$
\int_{0}^{\infty}\left\|P_{0} A_{0} A P_{0}\right\| d t<\infty
$$

$$
a_{n}(t)=\int_{t_{0}=0}^{t} \ldots \int_{t_{n}=0}^{t_{n}-1} P_{0} A_{0} P_{1} A_{1} P_{1} \ldots P_{1} A_{n} P_{1} A P_{0} d t_{n} \ldots d t_{0}
$$

suppose that for $n \geqq 1$

$$
\left\|a_{n}(t)\right\| \leqq c_{n} t^{n / 2}
$$

where the series $\sum_{n=1}^{\infty} c_{n} z^{n}$ has infinite radius of convergence. Suppose also that for some $\varepsilon>0, d_{n}$ and all $t \geqq 0$

$$
\left\|a_{n}(t)\right\| \leqq d_{n} t^{n / 2-\varepsilon} .
$$

Then the conditions of Theorem 2.1 are satisfied with

$$
K=\int_{0}^{\infty} P_{0} A_{0} A P_{0} d t_{0}
$$

Proof. Expanding $U_{t}^{\lambda}$ as a power series in $\lambda$ we obtain

$$
\|K(\lambda, \tau)-K\| \leqq \int_{\lambda=2_{\tau}}^{\infty}\left\|P_{0} A_{0} A P_{0}\right\| d t_{0}+\sum_{n=1}^{\infty} \lambda^{n}\left\|a_{n}(t)\right\| .
$$

By Eq. (2.36) the integral vanishes as $\lambda \rightarrow 0$ uniformly for $\tau$ in any compact subset of the open interval $(0, \infty)$. By Eq. $(2.38)$ if $0 \leqq \tau \leqq \tau_{0}$ the series is dominated by the convergent series $\sum_{n=1}^{\infty} c_{n} \tau_{0}^{n / 2}$. But by Eq. (2.39) the $n$th term of the series is also dominated by

$$
\lambda^{2 \varepsilon} d_{n} \tau_{0}^{n / 2-\varepsilon}
$$

which converges to zero as $\lambda \rightarrow 0$. Therefore the series converges to zero uniformly if $0 \leqq \tau \leqq \tau_{0}$.

The above theorems complete the abstract theory and we give some remarks and applications. 
(1) The Wigner-Weisskopf Atom. The solution in $[4,5]$ of the evolution equations for a harmonic oscillator weakly coupled to an infinite free heat bath reduces to a single-particle problem for the WignerWeisskopf atom. This and its multi-dimensional version [6], can be solved in a few lines using the above formalism. We remark, however, that the results obtained in $[4,6]$ are stronger than those given here in that the convergence was shown to be uniform in time. This is very important when discussing the interchange of the limits $\lambda \rightarrow 0$ and $t \rightarrow \infty$.

(2) Stochastic Differential Equations. There is a strong formal similarity of this work to a problem on stochastic differential equations on a Banach space $\mathscr{B}_{0}$. See [7]. If $(\Omega, d \omega)$ is a probability space then one can define

$$
\mathscr{B}=L^{1}\left(\Omega, \mathscr{B}_{0}, d \omega\right)
$$

and let the projection $P_{0}$ be

$$
P_{0} f=\int_{\Omega} f(\omega) d \omega .
$$

The interaction is then

$$
(A f)(\omega)=A(\omega) f(\omega)
$$

where $A(\omega)$ is a "random" operator-valued function, and the free evolution is

$$
\left(U_{t} f\right)(\omega)=f(t \omega)
$$

where $t \omega \in \Omega$ for all $t \in \mathbb{R}$ and $\omega \in \Omega$.

(3) On the Condition $\left\|P_{1}\right\|=1$. If the condition $\left\|P_{1}\right\|=1$ were satisfied than a lot of the technical trouble involved in verifying the conditions of Theorem 2.1 could be avoided. However in the example of the next section it may be seen that $\left\|P_{1}\right\|=2$. This difficulty also arose in [8] and was the reason for the condition on the spectrum of $(1-P) L(1-P)$ in Theorem 3.3 of that paper.

(4) Extensions of the Theorems. The theorems can be extended to certain cases where $\mathscr{B}_{0}$ is infinite dimensional and the operators involved are unbounded. However, a necessary restriction is that $Z_{0}$ has discrete spectrum, since otherwise the operation $\boxminus$ is not defined.

(5) The Origin of Irreversibility. We started with an evolution equation on $\mathscr{B}$ which is fully reversible and ended up with a semigroup on $\mathscr{B}_{0}$, which represents an irreversible dissipative process. The origin of the irreversibility in this case clearly lies in the initial conditions rather than in any dubious procedure such as coarse-graining. 


\section{§ 3. System in an Infinite Free Heat Bath}

We show that an $N$-level atom weakly coupled to an infinite free heat bath relaxes to equilibrium in a Markovian fashion and that the equilibrium state is its Gibbs state at the temperature of the heat bath. This behaviour has already been proved in two particular cases $[4,9]$. Our contribution is therefore to show that the result is of a very general type, being essentially independent of the nature of the system, and of whether the coupling to the bath is linear in the field operators (at least in the fermion cases). The problem could, as in [9], be solved without the use of master equations, but we believe they form a useful device for extracting the terms which contribute to the limiting behaviour. We make comments on possible variations of the model at the end of the section.

The atom is described by an $N$-dimensional Hilbert space $\mathscr{K}_{A}$ with a free Hamiltonian $H_{A}$. The heat bath is described by a quasi-free representation of the canonical anticommutation relations (CAR's) with an infinite number of degrees of freedom [10]. To be specific we let the complex Hilbert space $\mathscr{V}$ be the test function space and $S$ the single particle Hamiltonian on $\mathscr{V}$. For each $f \in \mathscr{V}$ we have a bounded operator $\varphi_{f}$ on a space $\mathscr{K}_{B}$ satisfying the CAR's

$$
\varphi_{f} \varphi_{g}+\varphi_{g} \varphi_{f}=2 \operatorname{Re}\langle f, g\rangle 1 .
$$

There is given a cyclic vector $\Omega$ in $\mathscr{K}_{B}$ and a Hamiltonian $H_{B}$ on $\mathscr{K}_{B}$ such that

and

$$
H_{B} \Omega=0
$$

$$
e^{i H_{B} t} \varphi(f) e^{-i H_{B} t}=\varphi\left(e^{i S t} f\right) .
$$

The representation is determined by its correlation functions as follows. For any integer $n$ we define the set $\mathscr{P}_{n}$ of pairings as the set of all permutations $p$ of $(1, \ldots, 2 n)$ such that

$$
p(2 r-1)<p(2 r) \text { and } \quad p(2 r-1)<p(2 r+1)
$$

for all $r$. Then writing $\langle\ldots\rangle$ for the expectation with respect to $\Omega$,

while

$$
\left\langle\varphi\left(f_{1}\right) \ldots \varphi\left(f_{2 n}\right)\right\rangle=\sum_{p \in \mathscr{P}_{n}} \operatorname{sign} p \prod_{r=1}^{n}\left\langle\varphi\left(f_{p(2 r-1)}\right) \varphi\left(f_{p(2 r)}\right)\right\rangle
$$

$$
\left\langle\varphi\left(f_{1}\right) \ldots \varphi\left(f_{2 n+1}\right)\right\rangle=0 .
$$


The formula for the two-point functions at the inverse temperature $\beta$ is

$$
\begin{aligned}
\langle\varphi(f) \varphi(g)\rangle_{\beta}=\langle f, g\rangle & +\left\langle\left(1+e^{-\beta S}\right)^{-1} g, f\right\rangle \\
& -\left\langle\left(1+e^{-\beta S}\right)^{-1} f, g\right\rangle .
\end{aligned}
$$

The Hilbert space for the composite system is $\mathscr{K}=\mathscr{K}_{A} \otimes \mathscr{K}_{B}$ and the Hamiltonian is

$$
H_{\lambda}=H_{A} \otimes 1+1 \otimes H_{B}+\lambda H_{I}
$$

where the interaction term is

$$
H_{I}=Q \otimes \Phi .
$$

Here $Q$ is an arbitrary self-adjoint operator on $\mathscr{K}_{A}$ and the self-adjoint operator $\Phi$ is given by

$$
\Phi=i \varphi\left(f_{1}\right) \varphi\left(f_{-1}\right)
$$

where the test functions $f_{1}$ and $f_{-1}$ are supposed to have disjoint energy spectra, that is

$$
\left\langle e^{i S t} f_{1}, f_{-1}\right\rangle=0
$$

for all $t \in \mathbb{R}$.

We now state the problem in the terminology of Section 2. Let the Banach space $\mathscr{B}$ be the space of trace class operators on $\mathscr{K}$ and let $\mathscr{B}_{0}$ be the space of trace class operators on $\mathscr{K}_{A}$. The projection $P_{0}: \mathscr{B} \rightarrow \mathscr{B}_{0}$ is the partial trace, which is determined by

$$
\operatorname{tr}\left[P_{0}(\varrho) X\right]=\operatorname{tr}[\varrho(X \otimes 1)]
$$

if $\varrho$ is an arbitrary trace class operator on $\mathscr{K}$ and $X$ is an arbitrary bounded operator on $\mathscr{K}_{A}$. For this to be a projection we have to identify $\mathscr{B}_{0}$ as a subspace of $\mathscr{B}$, and we do this by the injection $\varrho \rightarrow \varrho \otimes \sigma$ where $\sigma$ is the state $|\Omega\rangle\langle\Omega|$ on $\mathscr{K}_{B}$.

The free evolution is the one parameter group of isometries on $\mathscr{B}$ given by

$$
U_{t}(\varrho)=e^{-i H_{0} t} \varrho e^{i H_{0} t}
$$

whose infinitesimal generator is formally

$$
Z(\varrho)=-i\left[H_{0}, \varrho\right] \text {. }
$$

The necessary and sufficient condition for $U_{t}$ to leave $\mathscr{B}_{0}$ and $\mathscr{B}_{1}$ $=\left(1-P_{0}\right) \mathscr{B}$ invariant is that $H_{B} \Omega=0$, which we have assumed. The perturbation introduced is the derivation $A$ on $\mathscr{B}$ given by

$$
A(\varrho)=-i[Q \otimes \Phi, \varrho] \text {. }
$$


The evolution group of the total system is therefore

$$
V_{t}^{\lambda}(\varrho)=e^{-i H_{\lambda} t} \varrho e^{i H_{\lambda} t} .
$$

The condition $A_{00}=0$ is satisfied provided

$$
\langle\Phi \Omega, \Omega\rangle=0
$$

and this is a consequence of Eqs. (3.10) and (3.11).

We have now set up the problem in the notation of Section 2 and have to check that the conditions of Theorem 2.3 are satisfied. We first introduce the following abbreviated notation.

$$
\begin{gathered}
A_{r}=U_{-t_{r}} A U_{t_{r}}, \quad Q_{r}=e^{i H_{A} t_{r}} Q e^{-i H_{A} t_{r}} \\
\Phi_{r}=e^{i H_{B} t_{r}} \Phi e^{-i H_{B} t_{r}} \\
\varphi_{r}(f)=e^{i H_{B} t_{r}} \varphi(f) e^{-i H_{B} t_{r}}=\varphi\left(e^{i S t_{r}} f\right)
\end{gathered}
$$

and define

$$
\begin{aligned}
h(t) & =\left\langle e^{i H_{B} t} \Phi e^{-i H_{B} t} \Phi \Omega, \Omega\right\rangle \\
h_{i}(t) & =\left\langle e^{i H_{B} t} \varphi\left(f_{i}\right) e^{-i H_{B} t} \varphi\left(f_{i}\right) \Omega, \Omega\right\rangle .
\end{aligned}
$$

It may easily be proved from Eqs. (3.5) and (3.11) that

$$
h(t)=h_{1}(t) h_{-1}(t) .
$$

Theorem 3.1. If $\int_{0}^{\infty}\left|h_{1}(t)\right| d t<\infty$ then

$$
\int_{0}^{\infty}\left\|P_{0} A_{0} A P_{0}\right\| d t_{0}<\infty
$$

Proof. If $\varrho \in \mathscr{B}_{0}$ then

$$
\begin{aligned}
P_{0} A_{0} A P_{0} \varrho= & -P_{0}\left[Q_{0} \otimes \Phi_{0},[Q \otimes \Phi, \varrho \otimes \sigma]\right] \\
= & -Q_{0} Q \varrho \operatorname{tr}\left[\Phi_{0} \Phi \sigma\right]+Q_{0} \varrho Q \operatorname{tr}\left[\Phi_{0} \sigma \Phi\right] \\
& +Q \varrho Q_{0} \operatorname{tr}\left[\Phi \sigma \Phi_{0}\right]-\varrho Q Q_{0} \operatorname{tr}\left[\sigma \Phi \Phi_{0}\right] .
\end{aligned}
$$

Therefore

$$
\begin{aligned}
\left\|P_{0} A_{0} A P_{0}\right\| & \leqq 4\|Q\|^{2}\left|h\left(t_{0}\right)\right| \\
& \leqq 4\|Q\|^{2}\left\|f_{-1}\right\|^{2}\left|h_{1}\left(t_{0}\right)\right|
\end{aligned}
$$

which immediately yields the result.

Lemma 3.2. $a_{2 n+1}(t)=0$ for all $t \geqq 0$ and

$$
a_{2 n}(t)=\int_{t_{0}=0}^{t} \ldots \int_{t_{2 n}=0}^{t_{2 n-1}} P_{0} A_{0} A_{1} P_{1} A_{2} A_{3} P_{1} \ldots P_{1} A_{2 n} A P_{0} d t_{2 n} \ldots d t_{0}
$$


Proof. Let $N$ be the operator counting the number of particles whose energy lies in the energy spectrum of $f_{1}$, so that $\left[N, \varphi\left(f_{-1}\right)\right]=0$, and define $\mathscr{E}: \mathscr{B} \rightarrow \mathscr{B}$ by

$$
\mathscr{E}(\varrho)=\left(1 \otimes e^{\pi i N}\right) \varrho\left(1 \otimes e^{-\pi i N}\right) .
$$

Then for all $\varrho \in \mathscr{B}_{0}$ and all $t \geqq 0$

$$
\begin{aligned}
\mathscr{E} U_{t} & =U_{t} \mathscr{E}, \\
\mathscr{E} A & =-A \mathscr{E}, \quad \mathscr{E} P_{0}=P_{0} \mathscr{E} \\
\mathscr{E} & =\varrho
\end{aligned}
$$

The statements of the lemma follow by applying the symmetry $\mathscr{E}$ to the integrand.

In the following we shall occasionally write $A_{2 n+1}$ for $A$; this is equivalent to introducing a dummy variable $t_{2 n+1} \equiv 0$.

Lemma 3.3. Let $h_{1}$ be integrable on $[0, \infty]$. Then if $\pi$ is any permutation of $(0, \ldots, 2 n+1)$

$$
\begin{gathered}
\left|\sum_{p} \int_{t_{0}=0}^{t} \ldots \int_{t_{2 n}=0}^{t_{2 n-1}} \prod_{r=0}^{n} h_{1}\left(t_{\pi p(2 r)}-t_{\pi p(2 r+1)}\right) d t_{2 n} \ldots d t_{0}\right| \\
\leqq t^{n}\left\|h_{1}\right\|_{1}^{n+1} / 2^{n+1}(n+1) !
\end{gathered}
$$

Proof. For every permutation $\sigma$ of $(0, \ldots, 2 n+1)$ we obtain a pairing by associating $\sigma(2 r)$ with $\sigma(2 r+1)$ and then reordering appropriately. After counting repetitions this shows that the integral is dominated by

$$
\begin{aligned}
& \sum_{\sigma \in S_{2 n+2}} \frac{1}{2^{n+1}(n+1) !} \int_{t_{0}=0}^{t} \ldots \int_{t_{2 n}=0}^{t_{2 n-1}} \prod_{r=0}^{n} \mid h_{1}\left(t_{\sigma(r)}-t_{\sigma(2 r+1)} \mid d t_{2 n} \ldots d t_{0}\right. \\
& =\frac{1}{2^{n+1}(n+1) !} \int_{t_{0}=0}^{t} \ldots \int_{t_{2 n}=0}^{t} \prod_{r=0}^{n}\left|h_{1}\left(t_{2 r}-t_{2 r+1}\right)\right| d t_{2 n} \ldots d t_{0}
\end{aligned}
$$

since $\left|h_{1}\right|$ is an even function. Integrating with respect to the even variables and remembering that $t_{2 n+1} \equiv 0$, the result follows.

Theorem 3.4. If $\left\|h_{1}\right\|_{1}<\infty$ then Eq. (2.38) of Theorem 2.3 is satisfied for constants $c_{n}$ of the required type.

Proof. We put

$$
\Phi_{r}^{L}(\varrho)=\Phi_{r} \varrho, \quad \Phi_{r}^{R}(\varrho)=\varrho \Phi_{r}
$$

and similarly for $Q$ and $A$, so that

$$
A_{r}(\varrho)=\left(A_{r}^{L}+A_{r}^{R}\right)(\varrho)=\left(-i Q_{r}^{L} \Phi_{r}^{L}+i Q_{r}^{R} \Phi_{r}^{R}\right)(\varrho) .
$$


We now expand

$$
\begin{aligned}
a_{2 n}(t) \varrho= & \int_{t_{0}=0}^{t} \ldots \int_{t_{2 n}=0}^{t_{2 n-1}} P_{0}\left(A_{0}^{L}+A_{0}^{R}\right)\left(A_{1}^{L}+A_{1}^{R}\right)\left(1-P_{0}\right) \ldots \\
& \ldots\left(1-P_{0}\right)\left(A_{2 n}^{L}+A_{2 n}^{R}\right)\left(A^{L}+A^{R}\right) P_{0} \varrho d t_{2 n} \ldots d t_{0}
\end{aligned}
$$

to obtain

$$
\begin{gathered}
a_{2 n}(t) \varrho=\sum_{\alpha, \beta} \operatorname{sign} \alpha \int_{t_{0}=0}^{t} \ldots \int_{t_{2 n}=0}^{t_{2 n-1}} P_{0} A_{0}^{\beta(0)} A_{1}^{\beta(1)} A_{2}^{\beta(2)} \ldots \\
A_{\alpha_{1}-1}^{\beta\left(\alpha_{1}-1\right)} P_{0} A_{\alpha_{1}}^{\beta\left(\alpha_{1}\right)} \ldots A_{\alpha_{j}-1}^{\beta\left(\alpha_{j}-1\right)} P_{0} A_{\alpha_{j}}^{\beta\left(\alpha_{j}\right)} \ldots \\
\ldots A_{2 n}^{\beta(2 n)} A_{2 n+1}^{\beta(2 n+1)} P_{0} \varrho d t_{2 n} \ldots d t_{0}
\end{gathered}
$$

In this equation we sum over all functions $\beta:\{0, \ldots, 2 n+1\} \rightarrow\{L, R\}$. We sum over all sequences $\alpha(0), \ldots, \alpha(k+1)$ of even integers such that

$$
0=\alpha(0)<\alpha(1)<\cdots<\alpha(k)<\alpha(k+1)=2 n+2 .
$$

We have put $\operatorname{sign} \alpha=(-1)^{k}$ and have introduced the dummy variable $t_{2 n+1} \equiv 0$.

It may be observed that the operators in the above integral are tensor products and that $P_{0}$ acts only on the second component. This leads immediately to the estimate

$$
\begin{gathered}
\left\|a_{2 n}(t) \varrho\right\| \leqq \sum_{\alpha, \beta}\|Q\|^{2 n+2}\|\varrho\| \int_{t_{0}=0}^{t} \ldots \int_{t_{2 n}=0}^{t_{2 n-1}} d t_{2 n} \ldots d t_{0} . \\
\prod_{j=0}^{k}\left|\operatorname{tr}\left[\Phi_{\alpha_{j}}^{\beta\left(\alpha_{j}\right)} \Phi_{\alpha_{j}+1}^{\beta\left(\alpha_{j}+1\right)} \ldots \Phi_{\alpha_{j+1}-1}^{\beta\left(\alpha_{j+1}-1\right)} \sigma\right]\right| \\
=\sum_{\alpha, \beta}\|Q\|^{2 n+2}\|\varrho\| \int_{t_{0}=0}^{t} \ldots \int_{t_{2 n}=0}^{t_{2 n-1}} I\left(\alpha, \pi, t_{0} \ldots t_{2 n}\right) d t_{2 n} \ldots d t_{0}
\end{gathered}
$$

where

$$
I\left(\alpha, \pi, t_{0} \ldots t_{2 n}\right)=\prod_{j=0}^{k}\left|\left\langle\Phi_{\pi\left(\alpha_{j}\right)} \ldots \Phi_{\pi\left(\alpha_{j+1}-1\right)}\right\rangle\right|
$$

and the permutation $\pi$ of $\{0, \ldots, 2 n+1\}$ depends on $\alpha$ and $\beta$.

We now use the quasi-free hypothesis (for the first time) and Eq. (3.11) to deduce that

$$
\begin{aligned}
\left|I\left(\alpha, \pi, t_{0} \ldots t_{2 n}\right)\right| & \leqq\left\|f_{-1}\right\|^{2 n+2} \prod_{j=0}^{k}\left|\left\langle\varphi_{\pi\left(\alpha_{j}\right)}\left(f_{1}\right) \ldots \varphi_{\pi\left(\alpha_{j+1}-1\right)}\left(f_{1}\right)\right\rangle\right| \\
& \leqq\left\|f_{-1}\right\|^{2 n+2} \sum_{p} \prod_{r=0}^{n}\left|\left\langle\varphi_{\pi p(2 r)}\left(f_{1}\right) \varphi_{\pi p(2 r+1)}\left(f_{1}\right)\right\rangle\right|
\end{aligned}
$$


The use of Lemma 3.3 now yields

$$
\begin{aligned}
\left\|a_{2 n}(t)\right\| & \leqq \sum_{\alpha, \beta}\|Q\|^{2 n+2}\left\|f_{-1}\right\|^{2 n+2} t^{n}\left\|h_{1}\right\|_{1}^{n+1} / 2^{n+1}(n+1) ! \\
& \leqq 2^{2 n+1}\|Q\|^{2 n+2}\left\|f_{-1}\right\|^{2 n+2} t^{n}\left\|h_{1}\right\|_{1}^{n+1} /(n+1) !
\end{aligned}
$$

which is the required estimate.

Theorem 3.5. Suppose that for $i=1,2$ and some $\varepsilon>0$

$$
\int_{0}^{\infty}\left|h_{i}(t)\right|(1+|t|)^{\varepsilon} d t<\infty
$$

Then Eq. (2.39) of Theorem 2.3 is satisfied.

Proof. This is harder than Theorem 3.4 in that we must make use of some cancellations of the situation, but easier in that we do not need to control the dependence of $d_{n}$ on $n$.

We replace Eq. (3.31) by

$$
\begin{aligned}
a_{2 n}(t) \varrho= & \sum_{\alpha, \beta} \operatorname{sign} \alpha \int_{t_{0}=0}^{t} \ldots \int_{t_{2 n}=0}^{t_{2 n}-1} P_{0} A_{0}^{\beta(0)} A_{1}^{\beta(1)} A_{2}^{\beta(2)} \ldots \\
& \ldots A_{\alpha_{1}-1}^{\beta\left(\alpha_{1}-1\right)} P_{0} A_{\alpha_{1}}^{\beta\left(\alpha_{1}\right)} \ldots A_{\alpha_{k}-1}^{\beta\left(\alpha_{k}-1\right)} P_{0}\left\{A_{\alpha_{k}}^{\beta\left(\alpha_{k}\right)} A_{\alpha_{k}+1}^{\beta\left(\alpha_{k}+1\right)} \ldots A_{2 n+1}^{\beta(2 n+1)} P_{0} \varrho\right. \\
& \left.-A_{\alpha_{k}}^{\beta\left(\alpha_{k}\right)} A_{\alpha_{k}+1}^{\beta\left(\alpha_{k}+1\right)} \ldots A_{2 n-1}^{\beta(2 n-1)} P_{0} A_{2 n}^{\beta(2 n)} A_{2 n+1}^{\beta(2 n+1)} P_{0} \varrho\right\} \\
& \cdot d t_{2 n} \ldots d t_{0} .
\end{aligned}
$$

In this equation $\beta$ is as before but we sum over all sequences $\alpha(0), \ldots, \alpha(k)$ of even integers such that

$$
0=\alpha(0)<\alpha(1)<\cdots<\alpha(k)<2 n .
$$

As before this leads to the estimate

$$
\begin{aligned}
& \left\|a_{2 n}(t) \varrho\right\| \leqq \sum_{\alpha, \beta}\|Q\|^{2 n+2}\|\varrho\| \int_{t_{0}=0}^{t} \ldots \int_{t_{2 n}=0}^{t_{2 n-1}} d t_{2 n} \ldots d t_{0} . \\
& \prod_{j=0}^{k-1}\left|\operatorname{tr}\left[\Phi_{\alpha_{j}}^{\beta\left(\alpha_{j}\right)} \Phi_{\alpha_{j}+1}^{\beta\left(\alpha_{j}+1\right)} \ldots \Phi_{\alpha_{j+1}-1}^{\beta\left(\alpha_{j+1}-1\right)} \sigma\right]\right| . \\
& \mid \operatorname{tr}\left[\Phi_{\alpha_{k}}^{\beta\left(\alpha_{k}\right)} \Phi_{\alpha_{k}+1}^{\beta\left(\alpha_{k}+1\right)} \ldots \Phi_{2 n+1}^{\beta(2 n+1)} \sigma\right] \\
& -\operatorname{tr}\left[\Phi_{\alpha_{k}}^{\beta\left(\alpha_{k}\right)} \Phi_{\alpha_{k}+1}^{\beta\left(\alpha_{k}+1\right)} \ldots \Phi_{2 n-1}^{\beta(2 n-1)} \sigma\right] \operatorname{tr}\left[\Phi_{2 n}^{\beta(2 n)} \Phi_{2 n+1}^{\beta(2 n+1)} \sigma\right] \mid .
\end{aligned}
$$


Using the quasi-free hypothesis and Eq. (3.11) the last term of Eq. (3.42) can be written as

$$
\begin{aligned}
& J\left(\beta, t_{k}, t_{k+1}, \ldots, t_{2 n}\right) \\
& =\mid \prod_{i= \pm 1} \operatorname{tr}\left[\varphi_{\alpha_{k}}^{\beta\left(\alpha_{k}\right)}\left(f_{i}\right) \varphi_{\alpha_{k}+1}^{\beta\left(\alpha_{k}+1\right)}\left(f_{i}\right) \ldots \varphi_{2 n+1}^{\beta(2 n+1)}\left(f_{i}\right) \sigma\right] \\
& \quad-\prod_{i= \pm 1} \operatorname{tr}\left[\varphi_{\alpha_{k}}^{\beta\left(\alpha_{k}\right)}\left(f_{i}\right) \ldots \varphi_{2 n-1}^{\beta(2 n+1)}\left(f_{i}\right) \sigma\right] \operatorname{tr}\left[\varphi_{2 n}^{\beta(2 n)}\left(f_{i}\right) \varphi_{2 n+1}^{\beta(2 n+1)}\left(f_{i}\right) \sigma\right] \\
& =\left|a_{1} a_{-1}-b_{1} c_{1} b_{-1} c_{-1}\right|
\end{aligned}
$$

in an obvious notation,

$\leqq\left|a_{1}-b_{1} c_{1}\right|\left|a_{-1}\right|+\left|b_{1} c_{1}\right|\left|a_{-1}-b_{-1} c_{-1}\right|$.

Now again using the quasi-free hypothesis

$$
\begin{aligned}
J\left(\beta, t_{k}, \ldots, t_{2 n}\right) \leqq & \sum_{i=+1}\left\|f_{-i}\right\|^{2 n+2-\alpha_{k}} \sum_{p}^{\prime} \prod_{r=\frac{1}{2} \alpha_{k}}^{n} \\
& \cdot\left|\left\langle\varphi_{p(2 r)}\left(f_{i}\right) \varphi_{p(2 r+1)}\left(f_{i}\right)\right\rangle\right|
\end{aligned}
$$

where $\Sigma_{p}^{\prime}$ indicates the sum over all pairings $p$ of $\left\{\alpha_{k}, \alpha_{k}+1, \ldots, 2 n+1\right\}$ such that $2 n$ is not paired with $(2 n+1)$. Therefore

$$
\begin{aligned}
& \left\|a_{2 n}(t)\right\| \leqq \sum_{\alpha, \beta} \sum_{i=+1} \sum_{p}^{\prime \prime}\|Q\|^{2 n+2}\left\|f_{-i}\right\|^{2 n+2} \\
& \int_{t_{0}=0}^{t} \ldots \int_{t_{2 n}=0}^{t_{2 n-1}} \prod_{r=0}^{n}\left|h_{i}\left(t_{p(2 r)}-t_{p(2 r+1)}\right)\right| d t_{2 n} \ldots d t_{0}
\end{aligned}
$$

where $\Sigma_{p}^{\prime \prime}$ indicates the sum over all pairings of $\{0, \ldots, 2 n+1\}$ such that $2 n$ is not paired with $(2 n+1)$. Carrying out one-half of the integrations we see that each integral is dominated by an expression of the form

$$
\left\|h_{i}\right\|_{1}^{n} \int_{s_{0}=0}^{t} \ldots \int_{s_{n}=0}^{s_{n}-1}\left|h_{i}\left(s_{k}\right)\right| d s_{n} \ldots d s_{0}
$$

where $1 \leqq k \leqq n$,

$$
\begin{aligned}
& \leqq \text { const. } \int_{s=0}^{t}(t-s)^{n-k}\left|h_{i}(s)\right| s^{k} d s \\
& \leqq \text { const. } t^{n-\varepsilon} \int_{s=0}^{t} s^{\varepsilon}\left|h_{i}(s)\right| \mathrm{d} s .
\end{aligned}
$$

Together with Eq. (3.39) this proves the required estimate. 
This completes the proof of all the estimates required for the application of Theorem 2.3 to this model. We conclude this section with some comments on possible variations of the model. The interaction can be changed by putting

$$
H_{I}=Q \otimes \varphi\left(f_{1}\right) \ldots \varphi\left(f_{n}\right) i^{n(n-1) / 2}
$$

provided the test functions $f_{1}, \ldots, f_{n} \in \mathscr{V}$ satisfy

$$
\left\langle e^{i S t} f_{r}, f_{s}\right\rangle=0
$$

for all $r \neq s$ and all $t \neq 0$. In the more singular Boson case, however, one seems to be restricted to the case $n=1$, or possibly $n=2$, because of the difficulty of even proving the Hamiltonian is a self-adjoint operator. The proof can be extended to the case where the system is coupled to a finite number of heat baths at different temperatures. The space $\mathscr{K}_{A}$ need not be finite-dimensional provided $Q$ is bounded and $H_{A}$ has purely discrete spectrum. The theory can be developed in an algebraic form, as in [11], without any essential changes.

\section{§4. Dynamics of the Limit System}

We have shown that the model of the last section satisfies all the conditions of Theorem 2.3 provided Eq. (3.39) is satisfied. The operator $K^{\natural}$ on the space $\mathscr{B}_{0}$ of density matrices on $\mathscr{K}_{A}$ is given explicitly by

$$
\begin{aligned}
K^{\natural}(\varrho)= & \lim _{a \rightarrow \infty} \frac{1}{2 a} \int_{s=-a}^{a} \int_{t=0}^{\infty}\left\{-Q_{t+s} Q_{s} \varrho h(t)\right. \\
& \left.+Q_{t+s} \varrho Q_{s} \overline{h(t)}+Q_{s} \varrho Q_{t+s} h(t)-\varrho Q_{s} Q_{t+s} \overline{h(t)}\right\} d t d s
\end{aligned}
$$

where

$$
Q_{t}=e^{i H_{A} t} Q e^{-i H_{A} t}
$$

and $h(t)$ is defined by Eq. (3.19).

Lemma 4.1. The function $h(t)$ is continuous and integrable, and its transform satisfies

$$
\hat{h}(-x)=e^{-\beta x} \hat{h}(x)
$$

for all $x \in \mathbb{R}$, where $\beta$ is the inverse temperature of the heat bath. 
Proof. The integrability of $h(t)$ follows from Eqs. (3.20) and (3.39). By Eq. (3.7)

$$
\begin{aligned}
h_{i}(t)= & \left\langle e^{i S t} f_{i}, f_{i}\right\rangle+\left\langle\left(1+e^{-\beta S}\right)^{-1} f_{i}, e^{i S t} f_{i}\right\rangle \\
& -\left\langle\left(1+e^{-\beta S}\right)^{-1} e^{i S t} f_{i}, f_{i}\right\rangle
\end{aligned}
$$

so by the spectral theorem

$$
\hat{h}_{i}(-x)=e^{-\beta x} h_{i}(x) .
$$

Now by Eq. (3.20) and Fourier analysis

$$
\hat{h}(x)=\int_{-\infty}^{\infty} \hat{h}_{1}(y) \hat{h}_{-1}(x-y) d y
$$

which together with Eq. (4.5) yields Eq. (4.3).

We now expand

$$
Q_{t} \equiv e^{i H_{A} t} Q e^{-i H_{A} t}=\sum_{\omega} A_{\omega} e^{-i \omega t}
$$

so that the operator $A_{\omega}$ on $\mathscr{K}_{A}$ is zero unless $\omega$ is the difference of two eigenvalues of $H_{A}$.

Theorem 4.2. There exist real constants $a(\omega), e(\omega)$, and $s(\omega)$ satisfying

such that

$$
a(\omega)=e^{-\beta \omega} e(\omega) \geqq 0
$$

$$
\begin{aligned}
K^{\natural}(\varrho)= & \sum_{\omega \geq 0} e(\omega)\left\{-\frac{1}{2} A_{-\omega} A_{\omega} \varrho+A_{\omega} \varrho A_{-\omega}-\frac{1}{2} \varrho A_{-\omega} A_{\omega}\right\} \\
& +\sum_{\omega>0} a(\omega)\left\{-\frac{1}{2} A_{\omega} A_{-\omega} \varrho+A_{-\omega} \varrho A_{\omega}-\frac{1}{2} \varrho A_{\omega} A_{-\omega}\right\} \\
& +\sum_{\omega \in \mathbb{R}} \text { is }(\omega)\left[A_{-\omega} A_{\omega}, \varrho\right] .
\end{aligned}
$$

Comment. In the standard description of atomic radiation the three terms are respectively the emission term, the absorption term and a term describing a shift of the free energy levels (of order $\lambda^{2}$ ).

Proof. Substituting the expression for $Q_{t}$ in the definition of $K^{\natural}$ and evaluating the integral with respect to $x$ leads to

Now

$$
\begin{aligned}
\left.K^{\natural} \varrho\right)= & \sum_{\omega} \int_{t=0}^{\infty}\left\{-A_{\omega} A_{-\omega} \varrho e^{-i \omega t} h(t)\right. \\
& +A_{\omega} \varrho A_{-\omega} e^{-i \omega t} \overline{h(t)}+A_{-\omega} \varrho A_{\omega} e^{-i \omega t} h(t) \\
& \left.-\varrho A_{-\omega} A_{\omega} e^{-i \omega t} \overline{h(t)}\right\} d t .
\end{aligned}
$$

$$
\int_{0}^{\infty} h(t) e^{i \omega t} d t=\frac{1}{2} \hat{h}(\omega)+i s(\omega)
$$


where $\hat{h}(\omega)$ and $s(\omega)$ are real, so

$$
\begin{aligned}
K^{\natural}(\varrho)= & \frac{1}{2} \sum_{\omega}\left\{-A_{-\omega} A_{\omega} \varrho(\hat{h}(\omega)+2 i s(\omega))\right. \\
& +A_{\omega} \varrho A_{-\omega}(\hat{h}(\omega)-2 i s(\omega)) \\
& +A_{\omega} \varrho A_{-\omega}(\hat{h}(\omega)+2 i s(\omega)) \\
& \left.-\varrho A_{-\omega} A_{\omega}(\hat{h}(\omega)-2 i s(-\omega))\right\}
\end{aligned}
$$

which is the required result if for $\omega \geqq 0$

$$
e(\omega)=\hat{h}(\omega) \text { and } a(\omega)=\hat{h}(-\omega) .
$$

Theorem 4.3. The operator $\exp \left(K^{\natural} \tau\right)$ is for $\tau \geqq 0$ a positivity and trace preserving semigroup on $\mathscr{B}_{0}$.

Proof. This result is true because of the limiting procedure we used to obtain $K^{\natural}$, but we give an independent proof.

The formula

$$
\operatorname{tr}\left[\exp \left(K^{\natural} \tau\right) \varrho\right]=\operatorname{tr}[\varrho]
$$

for all $\tau \geqq 0$ is equivalent to

$$
\operatorname{tr}\left[K^{\natural} \varrho\right]=0
$$

for all $\varrho$, which is valid by inspection. The positivity will follow by the Trotter product formula [12] if we can write $K^{\natural}$ as a sum of generators of semigroups which preserve positivity. Now if $K_{1}(\varrho)=A \varrho A^{*}$ and $\varrho \geqq 0$ then

$$
e^{K_{1} \tau}(\varrho)=\Sigma \frac{\tau^{n}}{n !} A^{n} \varrho A^{* n} \geqq 0
$$

If $K_{2}(\varrho)=-A^{*} A \varrho-\varrho A^{*} A$ and $\varrho \geqq 0$ then

$$
e^{K_{2} \tau}(\varrho)=e^{-A^{*} A \tau} \varrho e^{-A^{*} A \tau} \geqq 0
$$

while if $H_{3}(\varrho)=i\left[A^{*} A, \varrho\right]$ and $\varrho \geqq 0$ then

$$
e^{K_{3} \tau}(\varrho)=e^{i A^{*} A \tau} \varrho^{-i A^{*} A \tau} \geqq 0 .
$$

This completes the proof.

The above results give a complete justification for regarding the equation

$$
\frac{d \varrho}{d \tau}=K^{\natural} \varrho
$$

as a quantum-mechanical Fokker-Planck equation. We draw the reader's attention to [13], where more general equations of this type have been studied from the point of view of quantum stochastic processes. In [14] it is shown that semigroups of this type can always be regarded as 
arising from interactions with singular heat baths, instead of from regular heat baths in the weak coupling limit. This is the point of view taken by Hepp and Lieb in [15]. They however work in the Heisenberg picture, which has some advantages.

We finally use the above equations to investigate the question of convergence to equilibrium. For the sake of simplicity we let $u_{1}, \ldots, u_{n}$ be an orthonormal basis of $\mathscr{K}_{A}$ and suppose

$$
H_{A} u_{r}=\omega_{r} u_{r}
$$

where the $\omega_{r}$ are all different.

Theorem 4.4. The semigroup $\exp \left\{K^{\natural} \tau\right\}$ on $\mathscr{B}_{0}$ leaves the subspace $\mathscr{D}$ of diagonal matrices invariant and defines a classical Markov process on the integers $\{1, \ldots, n\}$.

Proof. We first observe that

$$
\mathscr{D}=\left\{\varrho \in \mathscr{B}_{0}: U_{t} \varrho=\varrho \text { for all } t \in \mathbb{R}\right\} .
$$

If $\varrho \in \mathscr{D}$ then by Eq. (2.22)

$$
U_{t}\left(K^{\natural} \varrho\right)=K^{\natural} U_{t} \varrho=K^{\natural} \varrho
$$

so $K^{\natural} \varrho \in \mathscr{D}$. Therefore

Now put

$$
\exp \left\{K^{\natural} \tau\right\} \varrho=\sum_{n=0}^{\infty} K^{\natural n} \varrho \tau^{n} / n ! \in \mathscr{D} .
$$

$$
A_{r s}=\left\langle A u_{r}, u_{s}\right\rangle
$$

and define $v_{r} \in \mathscr{D}$ by

$$
v_{r}\left(u_{s}\right)=\delta_{r s} u_{s} .
$$

Then

$$
\begin{aligned}
K^{\natural}\left(v_{r}\right)= & \int_{t=0}^{\infty}\left\{-\sum_{s} e^{-i \omega_{r} t} A_{r s} e^{i \omega_{s} t} A_{s r} v_{r} h(t)\right. \\
& +\sum_{s} e^{-i \omega_{r} t} A_{s r} e^{i \omega_{r} t} A_{r s} v_{s} h(-t) \\
& +\sum_{s} A_{s r} e^{-i \omega_{r} t} A_{r s} e^{i \omega_{s} t} v_{s} h(t) \\
& \left.-\sum_{s} A_{r s} e^{-i \omega_{s} t} A_{s r} e^{i \omega_{r} t} v_{r} h(-t)\right\} d t \\
= & -\sum_{s} A_{r s} A_{s r} \hat{h}\left(m_{r}-\omega_{s}\right) v_{r} \\
& +\sum_{s} A_{s r} A_{r s} \hat{h}\left(m_{r}-\omega_{s}\right) v_{s} .
\end{aligned}
$$


Therefore

where

$$
K^{\natural}\left(v_{r}\right)=\sum_{s} a_{r s} v_{s}-\left(\sum_{s} a_{r s}\right) v_{r}
$$

$$
a_{r s}=A_{r s} A_{s r} \hat{h}\left(m_{r}-\omega_{s}\right) \geqq 0 .
$$

Identifying the diagonal matrices on $\mathscr{K}_{A}$ with the functions on $\{1, \ldots, n\}$ by

$$
\left\{\lambda_{1}, \ldots, \lambda_{n}\right\} \leftrightarrow \sum_{r=1}^{n} \lambda_{r} v_{r}
$$

the density matrices on $\mathscr{K}_{A}$ correspond to the probability measures on $\{1, \ldots, n\} . K^{\natural}$ is then the generator of a Markov semigroup on $\{1, \ldots, n\}$. If the process is ergodic (which certainly occurs if $a_{r s}>0$ for all $r, s$ ) then there is a unique equilibrium state and every state converges to the equilibrium state as $\tau \rightarrow \infty$. However ergodicity is not necessary for the existence of an equilibrium state.

Theorem 4.5. If the heat bath is at the inverse temperature $\beta$ then

$$
\varrho_{\beta}=\sum_{r=1}^{n} e^{-\beta \omega_{r}} v_{r} / \sum_{r=1}^{n} e^{-\beta \omega_{r}}
$$

is an equilibrium state for the Markov process on $\{1, \ldots, n\}$.

Proof. We have to show that

$$
0=K^{\natural} \varrho_{\beta}=\sum_{r, s} e^{-\beta \omega_{r}} a_{r s} v_{s}-\sum_{r, s} a_{r s} e^{-\beta \omega_{r}} v_{r}
$$

which is equivalent to

$$
\sum_{r} e^{-\beta \omega_{r}} a_{r s}=\sum_{r} a_{s r} e^{-\beta \omega_{s}} .
$$

This follows from Eqs. (4.3) and (4.29).

\section{References}

1. Prigogine, I.: The statistical interpretation of nonequilibrium entropy. In: Thirring, W., Cohen,E.G.D. (Eds.): The Boltzmann equation, theory and applications. BerlinHeidelberg-New York: Springer 1973

2. Haake,F.: Statistical treatment of open systems by generalised master equations. Springer tracts in modern physics, Vol. 66. Berlin-Heidelberg-New York: Springer 1973

3. Kato, T.: Perturbation theory for linear operators. Berlin-Heidelberg-New York: Springer 1966

4. Davies, E. B.: Commun. math. Phys. 33, 171-186 (1973)

5. Sewell, G. L.: Relaxation, amplification and the K.M.S. conditions (to appear)

6. Davies, E. B.: Dynamics of a multi-level Wigner-Weisskopf atom. J. math. Phys. (To appear) 
7. Papanicolaou, G. C., Varadhan, S. R. S.: Comm. Pure. Appl. Math. 26, 497-524 (1973)

8. Bongaarts, P.J.M., Fannes, M., Verbeure,A.: A remark on ergodicity, dissipativity, return to equilibrium, (To appear)

9. Pulè,J.V.: The Bloch equation. Commun. Math. Phys. (To appear) (1974)

10. Balslev, E., Verbeure,A.: Commun. math. Phys. 7, 55-76 (1968)

11. Presutti, E., Scacciatelli, E., Sewell, G. L., Wanderlingh, F.: J. Math. Phys. 13, 1085-1098 (1972)

12. Chernoff, P.R.: J. Funct. Anal. 2 (1968) $238-242$

13. Davies, E. B.: Commun. math. Phys. 19, 83-105 (1970)

14. Davies, E. B.: Z. Wahrscheinlichkeitsth. 23, 261-273 (1972)

15. Hepp, K., Lieb,E.H.: Phase transitions in reservoir-driven open systems with applications to lasers and superconductors. Helv. Phys. Acta 46, 573-603 (1973)

Communicated by K. Hepp
E. B. Davies

Mathematical Institute

24-29 St. Giles

Oxford, England 